\title{
Building Efficient Spectrum-Agile Devices for Dummies
}

\author{
Eugene Chai, Kang G. Shin \\ The University of Michigan \\ \{zontar, kgshin\}@eecs.umich.edu
}

\author{
Jeongkeun Lee, Sung-Ju Lee, Raul Etkin \\ Hewlett-Packard Labs \\ \{jklee, sjlee, raul.etkin\}@hp.com
}

\begin{abstract}
Spectrum management and device coordination for Dynamic Spectrum Access (DSA) networks have received significant research attention. However, current wireless devices have yet to fully embrace DSA networks due to the difficulties in realizing spectrumagile communications. We address the practical hurdles and present solutions towards implementing DSA devices, answering an important question "what is a simple practical extension to current wireless devices that makes them spectrum-agile?" To this end, we propose RoDIN, a general per-frame spectrum-shaping protocol that has the following features to support DSA in commercial off-the-shelf (COTS) wireless devices: (a) direct manipulation of passband signals from COTS devices, (b) fast FPGA-based spectrum shaping, and (c) a novel preamble design for spectrum agreement. RODIN uses an FPGA-based spectrum shaper together with a preamble I-FOP to achieve per-frame spectrum shaping with a delay of under $10 \mu \mathrm{s}$.
\end{abstract}

\section{Categories and Subject Descriptors}

C.2.1 [Computer-Communication Networks]: Network Architecture and Design-Wireless Communications; C.2.2 [ComputerCommunication Networks]: Network Protocols

\section{General Terms}

Algorithms, Experimentation, Design, Measurement, Performance

\section{Keywords}

Software-defined radio, hybrid radio, spectrum agility, per-frame spectrum shaping, spectrum-agile preamble detection

\section{INTRODUCTION}

Dynamic spectrum access (DSA), or spectrum agility, has become a popular solution to the problem of spectrum scarcity in wireless networks [6]. New devices that are designed to use only

\footnotetext{
The work reported in this paper was supported in part by the NSF under
} Grant CNS-1160775

Permission to make digital or hard copies of all or part of this work for personal or classroom use is granted without fee provided that copies are not made or distributed for profit or commercial advantage and that copies bear this notice and the full citation on the first page. To copy otherwise, to republish, to post on servers or to redistribute to lists, requires prior specific permission and/or a fee.

MobiCom'12, August 22-26, 2012, Istanbul, Turkey.

Copyright 2012 ACM 978-1-4503-1159-5/12/08 ...\$15.00. a monolithic block of spectrum can no longer expect to increase throughput by simply increasing their bandwidth. In fact, the throughput of an $802.11 \mathrm{n}$ device operating at $40 \mathrm{MHz}$ can even be lower than its throughput at $20 \mathrm{MHz}$ when encountering a $20 \mathrm{MHz}$ interference from another $802.11 \mathrm{~g}$ or $802.11 \mathrm{n}$ device $[8,22]$. Numerous other studies $[12,18]$ have reported performance anomalies when rate or bandwidth is blindly increased in an attempt to wrest more throughput from an overcrowded spectrum. We can only expect such problems to compound with the introduction of 802.11ac that supports up to $160 \mathrm{MHz}$ bandwidth. While this example deals with WiFi networks for clarity in exposition, the infeasibility of enhancing throughput by merely increasing bandwidth is also prevalent in non-WiFi networks. For example, a study of GSM usage patterns [15] shows that a wideband device cannot operate within the GSM band without some form of spectrum agility.

However, despite this obvious problem and the list of well-studied solutions, building efficient spectrum-agile devices is still a challenge for two main reasons. First, the current crop of commercial wireless devices are ill suited for DSA networks as they are primarily designed to use static, monolithic spectra. For example, spectrum- and bandwidth-agile platforms, such as SampleWidth [5] and FLUID [20], all have channel-switch times on the order of milliseconds. Second, the protocol stack does not fully support spectrum-agile communications. As an example, consider 802.11n OFDM frames that are detected by exploiting the self-correlation property of the preamble. This approach fails if the preamble is spread out over a non-contiguous spectrum, or in the face of interference from narrower band devices. Non-contiguous OFDM (NC-OFDM) techniques can be applied, but synchronization can be performed if and only if the set of non-contiguous subcarriers is known at the receiver beforehand.

We argue that the key capability that is missing from current state-of-the-art radio hardware is per-frame spectrum shaping. This is an important functional primitive that allows a radio to adapt to challenging channel conditions at the smallest practical unit of transmission.

\subsection{Why Per-Frame Spectrum Shaping?}

WiFi Channels. 802.11 devices are known to suffer significant performance degradation due to narrowband interference [11]. The effects of narrowband interference include timing recovery failure, the automatic gain control (AGC) failure due to an unexpected introduction of interference energy, and Physical Layer Convergence Protocol (PLCP) header processing failure.

Rapid frequency hopping (FH) by an 802.11 device [11] has been shown to improve its performance in the presence of narrowband interference. However, $\mathrm{FH}$ cannot avoid interference from a $\mathrm{FH}$ interferer, such as Bluetooth, if the hopping sequences of the WiFi and the interferer are not properly synchronized. Furthermore, col- 
lisions between multiple $\mathrm{FH}$ devices using different hopping sequences is a well-known challenge when scaling $\mathrm{FH}$ to a larger network [17].

This disadvantage of $\mathrm{FH}$ comes from the fact that it switches channels blindly, even when there is no interference on the channel it is currently using. This increases the possibility of the FH itself interfering with devices on other channels. We posit that a reactive approach to interference avoidance using per-frame spectrum shaping will enable 802.11 devices to avoid narrowband interference while maintaining high throughput and manageability. The use of per-frame spectrum shaping effectively re-allocates the spectrum of a transmission dynamically only when interference is detected on the channel. This minimizes the amount of spectrum touched by an 802.11 device and avoids the unnecessary channel-switch overhead when no interference is detected.

Non-WiFi Channels. Devices operating in non-WiFi channels have to contend with severe spectrum fragmentation due to multiple narrowband interferers. We illustrate this using spectrum traces [29] that took measurements from a $1.5 \mathrm{GHz}$ band and is centered at $770 \mathrm{MHz}$ frequency. This trace set thus covers multiple GSM and TV channels.

Fig. 1 shows the availability and outage durations of 1,5 and $20 \mathrm{MHz}$ monolithic channels operating within this band. Consider, in particular, the $20 \mathrm{MHz}$ transmission that is typical of WiFi devices. At a first glance, the long median channel-availability duration of 3 s can easily accommodate the channel-switch time of typical WiFi devices. However, we observe from Fig. 2 that monolithic $20 \mathrm{MHz}$ channels can transmit only about $6 \%$ of the time. This low availability is due to the presence of multiple uncoordinated narrow bandwidth interferers. Hence, in order to sustain a $20 \mathrm{MHz}$ transmission, multiple discontiguous $1 \mathrm{MHz}$ (or narrower) channels have to be bonded together. Given that the correlation between the different channels is low [15], such a device can expect to continuously reconfigure its set of bonded channels to avoid primary user interference. The otherwise long outage duration that it faces, as shown in Fig. 1, will severely degrade the quality of service. The ability to perform per-frame spectrum shaping is thus key for operating in non-WiFi channels as well.

\subsection{The Limitation of SDRs}

Software-defined radios (SDRs) have been used to develop the flexible RF interfaces required for DSA devices. However, SDR platforms face problems arising from poor efficiency and high complexity. SDR platforms, such as USRP [27] and SORA [26], are limited by the efficiency of a general-purpose platform in multitasking real-time DSP with other system tasks, while FPGA-based SDR platforms, such as WARP [28], are complex to work with. This complexity and inefficiency poses a significant challenge because it is necessary to re-implement the entire MAC/PHY protocol on the SDR platform in order to reap the advantage of PHY-layer flexibility.

\subsection{The Limitation of COTS Devices}

A commercial off-the-shelf (COTS) device that has its RF frontend separated from the MAC baseband chipset can facilitate easy integration between the SDR and COTS. However, COTS devices are increasingly implemented as single-chip solutions to improve power and space efficiency. This limits the flexibility of the RF frontends of COTS devices in supporting the various spectrum management policies required for per-frame spectrum shaping.

\subsection{The Challenge}

We take a very different approach to DSA and address an im- portant question: "What is a simple practical extension to current wireless devices that makes them spectrum agile?" We stress that any solution must be general enough to apply to the majority of COTS wireless devices currently available, yet simple enough to minimize the additional overhead that are added to COTS devices.

The intuition behind this comes from the fact that neither COTS devices nor SDRs are individually capable of supporting the perframe spectrum shaping necessary for DSA. Hence, a hybrid platform built using both SDRs and COTS devices is necessary. The SDR handles only the necessary PHY-layer manipulations, while the COTS device handles the main MAC/PHY processing. A practical DSA extension must have the following three important properties.

Property 1: Protocol independence. It must support as many current wireless protocols as possible. Hence, a COTS device should only have to be "plugged into" a DSA extension platform to gain spectrum agility. In reality, some modifications to the COTS platform may be necessary, but such changes must be minimal. Easy deployability of a DSA extension platform will naturally maximize the chance of its widespread acceptance. With this property, RODIN can be easily integrated into both OFDM and non-OFDM COTS devices.

Property 2: Per-frame spectrum shaping. Per-frame spectrum shaping is a general spectrum-shaping primitive that can be used to construct other spectrum-management protocols. In the absence of detailed knowledge about the behavior of other devices in the ISM or whitespace bands, a DSA platform must be able to adjust its spectral use on a frame-by-frame basis to react to unexpected transmissions by primary users.

Property 3: Fast spectrum agreement. Besides having the capability of per-frame spectrum shaping, the transmitter and receiver(s) must also agree on a common set of (possibly non-contiguous) spectrum bands before commencing transmission. Prior work on spectrum agreement made use of control channels [31], pre-defined backup channel lists [23], or centralized channel assignment [20]. Unfortunately, these approaches are too slow to meet the required delay bounds for per-frame spectrum shaping.

\subsection{Rodin: Our Solution}

We propose RoDIN ${ }^{2}$ - a hardware DSA extension to COTS devices. RODIN consists of three key components that enable it to serve as a drop-in DSA extension to arbitrary wireless devices.

Direct connection to COTS device. RODIN connects to a COTS device directly through the antenna port(s) on the COTS radio, thus upgrading unmodified COTS devices with spectrum agility.

Fast FPGA-based spectrum shaping. RODIN can split the spectrum of an unmodified signal from the COTS device into multiple non-contiguous spectrum subbands; the individual subbands are transmitted on unoccupied portions of the spectrum to avoid interference from other narrowband transmitters. RoDIN does not decode the signals to and from the COTS device. Our hardware implementation achieves this spectrum subdivision of each frame within $2 \mu s$ of detecting a passband signal from the COTS device.

Novel preamble design for spectrum agreement. A RODIN transmitter uses a novel preamble design to notify a RODIN receiver of the spectrum occupied by the accompanying spectrally-reshaped frame. With this preamble, RoDIN eliminates the need for a separate control channel, backup channel lists or a centralized spectrum coordinator. This preamble, when combined with fast spectrum shaping, enables RODIN to rapidly adapt to any primary transmission pattern seen on channels.

\footnotetext{
${ }^{2}$ Named after Auguste Rodin, the French sculptor.
} 


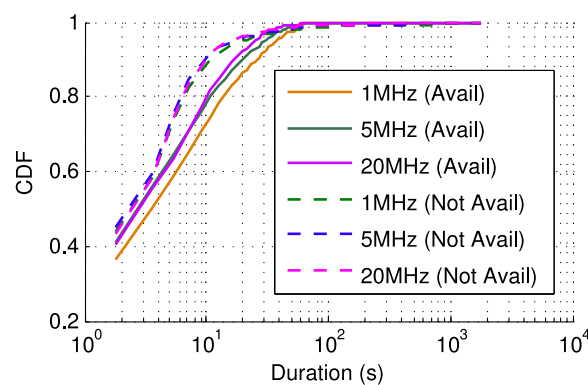

Figure 1: CDF of the channel busy and available durations.
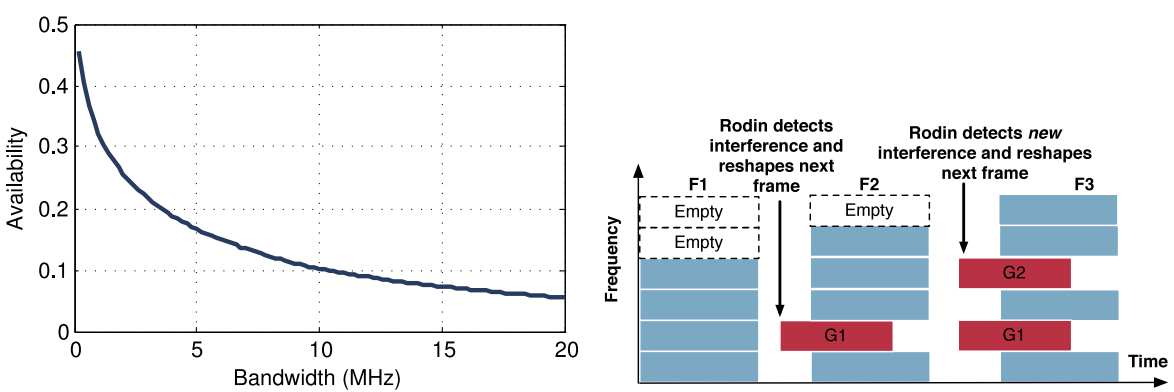

Figure 2: Channel availability of different Figure 3: Transmission of 3 frames $F_{1}, F_{2}$ transmission bandwidths. and $F_{3}$ using RODIN. RODIN reshapes the spectrum of $F_{2}$ and $F_{3}$ to avoid interference from $G_{1}$ and $G_{2}$, respectively.

To see how efficiently this can be done, consider shaping a $20 \mathrm{MHz}$ $802.11 \mathrm{n}$ frame over multiple $5 \mathrm{MHz}$ subbands. Spectrum agreement and shaping can be achieved in under $10 \mu \mathrm{s}$. This adds only $3.8 \%$ of additional overhead to the transmission time of an $802.11 \mathrm{n}$ frame without aggregation. The overhead will be even lower if frame aggregation is used. The negligible overhead enables RoDIN to react to rapidly changing channel conditions on all types of channels.

RODIN is a novel RF frontend for COTS devices for cognitive spectrum management. In the short term, it extends the experimental capabilities of COTS devices but it can also be built into COTS devices to achieve integrated SDR-COTS hybrids in the future.

Our contributions in this paper are: (a) a detailed design of spectrum shaping and agreement in RODIN, (b) an evaluation of the real-world performance of RODIN via controlled experiments with FPGA-based implementations, and (c) an analysis of the performance of RODIN using detailed channel measurements.

\section{OVERVIEW OF RODIN}

RODIN is a general-purpose per-frame spectrum-sculpting platform designed for wideband frame-based COTS devices. In particular,

- RODIN is designed for wideband COTS devices that share the spectrum with other devices of narrower bandwidth. Examples of such scenarios include $160 \mathrm{MHz} 802.11 \mathrm{ac}$ or $40 \mathrm{MHz}$ $802.11 \mathrm{n}$ devices that share the same $5 \mathrm{GHz}$ band with $802.11 \mathrm{a}$ devices operating at $20 \mathrm{MHz}$; UWB devices that share the spectrum with narrowband cellular networks.

- RODIN assumes that the maximum bandwidth of its SDR $\mathrm{RF}$ frontend is greater than the bandwidth of the transmitted COTS signal. RODIN shapes the spectrum of each frame while keeping the overall transmission bandwidth constant. Note that RODIN does not change the operating bandwidth of the COTS device.

- RODIN is designed for CSMA networks with multiple concurrent asynchronous transmitters that occupy non-overlapping spectra. This maximizes the frequency reuse of wireless channels. However, these channels are not perfectly orthogonal to each other due to non-ideal pulse shaping filters [16].

RODIN has three key features to function as a general per-frame spectrum-shaping platform for COTS devices: (a) capability for direct connection to the COTS device, (b) FPGA-based spectrum shaping, and (c) a novel preamble design for fast spectrum agreement.

RoDIN divides its total RF bandwidth $B$ into $N$ subbands and shapes the spectrum of a frame that occupies $N_{F}(<N)$ of these

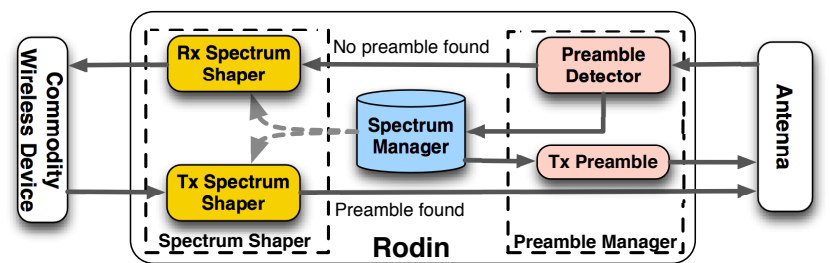

Figure 4: High-level architecture of RoDIN.

subbands. Fig. 3 shows an example of RODIN reshaping a wideband transmission, with $N=6$ and $N_{F}=4$, in the face of narrowband interference. Frame $F_{1}$ can be transmitted without any additional shaping since no interfering transmission is present. However, almost immediately after transmitting $F_{1}$, RODIN detects a narrowband interference $G_{1}$ that occupies one subband. It maps the spectrum of $F_{2}$ into the remaining subbands and transmits it without interfering with $G_{1}$. This frame-by-frame spectrum reshaping is repeated for $F_{3}$ to avoid interference from $G_{2}$.

If per-frame spectrum shaping is not used, a wideband transmission would be blocked by a narrowband transmission, or a wideband transmission collides with a narrowband transmission if the narrowband transmitter does not correctly detect the wideband transmission.

These features are realized with the system architecture shown in Fig. 4. The Spectrum Shaper reshapes the signal to and from the COTS wireless device in real time, while the Preamble Manager, consisting of a preamble detector and a preamble constructor, uses specially-constructed preambles to exchange spectrum information between RoDIN devices. The Spectrum Manager executes a protocol that selects the best set of spectrum bands for a particular transmitter-receiver pair.

These components are detailed in the rest of this paper. For simplicity, our current design of RODIN is limited to SISO devices only, although an extension to MIMO devices is straightforward.

\section{SPECTRUM SHAPING IN RODIN}

Spectrum shaping divides the spectrum occupied by a COTS device into multiple discontiguous frequency bands. In order to realize real-time spectrum shaping, (a) the spectrum-shaping procedure must have low latency and (b) the spectrum shapers on the transmitter and the receiver must cooperate with minimal synchronization. Property (a) relates to the efficiency of the spectrum shaper - upon specification of the desired subbands, the shaper must quickly re- 
shape the spectrum with minimal delay. In contrast, property (b) relates to the tolerance of the spectrum shaper to errors caused by channel distortion, timing, frequency shifts, etc. This is particularly important since different PHY protocols engage different measures to combat distortions. For example, DSSS-based protocols use Rake receivers and equalizers while OFDM-based protocols use the Schmidl-Cox algorithm. Obviously, it is not feasible for RoDIN to support the wide variety of synchronization primitives to achieve protocol independence. Hence, RODIN focuses on spectrum shaping while leaving protocol-specific DSP functions (such as pilot handling) to the COTS device.

In the rest of this section, we only describe a two-band shaping process $\left(N>N_{F}=2\right)$ for the sake of clarity. This process can be easily extended to multi-band shaping.

\subsection{Overview of Spectrum Shaping}

Let $X(f)$ denote the original spectrum of the frame received by RODIN from the attached wireless device. The spectrum-shaping procedure for the frame transmission consists of the following components.

(a) Pre-filter modulation. RODIN only uses low-pass filters for spectrum shaping. Hence, the input signal $X(f)$ must be modulated to align the relevant portion of $X(f)$ with the passband of the filter $H(f)$. Let $m_{1}^{(a)}(t)=\exp \left\{j 2 \pi k_{1} B t / N\right\}$ and $m_{2}^{(a)}(t)=$ $\exp \left\{j 2 \pi k_{2} B t / N\right\}$ be the time-domain complex-valued carrier used to modulate $X(f)$, with $k_{i}=0, \ldots, N-1, \forall i=1,2$. The modulated spectrum is:

$$
\begin{aligned}
X_{i}^{(a)}(f) & =X(f) * \delta\left(f-k_{i} B / N\right) \\
& =X\left(f-k_{i} B / N\right), \forall i=1,2
\end{aligned}
$$

where $\delta(\cdot)$ is the Dirac delta function.

(b) Filtering. Once the spectrum of the input signal has been appropriately modulated, a low-pass filter is applied to split the input spectrum into two separate subbands. Let $H_{1}(f)$ and $H_{2}(f)$ be the two low-pass filters used in this example. The two spectral subbands $X_{1}^{(b)}(f)$ and $X_{2}^{(b)}(f)$ are:

$$
\begin{aligned}
X_{i}^{(b)}(f) & =H_{i}(f) X_{i}^{(a)}(f) \\
& =H_{i}(f) X\left(f-k_{i} B / N\right), \forall i=1,2
\end{aligned}
$$

(c) Post-filter modulation. Each filtered subband must be transmitted at a frequency that encounters minimum interference. This modulation step uses $m_{1}^{(c)}(t)=\exp \left\{j 2 \pi l_{1} B t / N\right\}$ and $m_{2}^{(c)}(t)=$ $\exp \left\{j 2 \pi l_{2} B t / N\right\}$ as the modulating carrier, where $l_{1}, l_{2}=1, \ldots, N$. The second modulation step achieves, $\forall i=1,2$ :

$$
\begin{aligned}
X_{i}^{(c)}(f) & =X_{i}^{(b)}(f) * \delta\left(f-l_{i} B / N\right)=X_{i}^{(b)}\left(f-l_{i} B / N\right) \\
& =H_{i}\left(f-l_{i} B / N\right) X\left(f-\left(l_{i}+k_{i}\right) B / N\right)
\end{aligned}
$$

(d) Combining spectra. Finally, the two subbands are added to produce a single spectrally non-contiguous frame. This results in a single time-domain data stream that is sent to the radio frontend of RODIN to be transmitted:

$$
Y(f)=X_{1}^{(c)}(f)+X_{2}^{(c)}(f) .
$$

The RODIN receiver executes the same process as shown in Fig. 5 using the same low-pass filters but with the modulation sinusoids rearranged as:

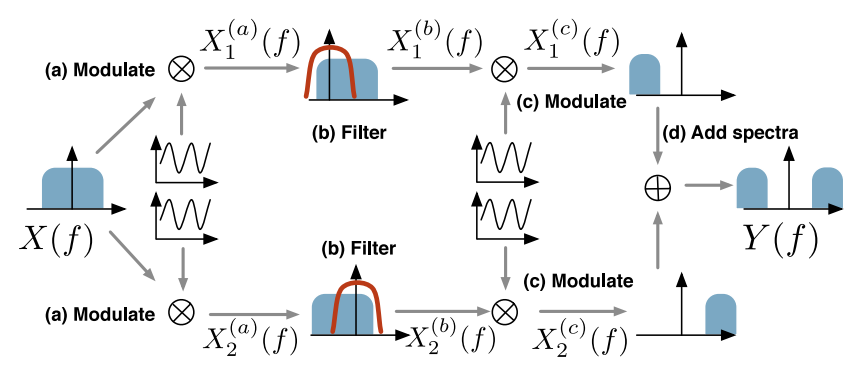

Figure 5: Shaping a frame occupying a contiguous spectrum $X(f)$ into two separate spectrum bands $Y(f)$. The shaping procedure is a 4-step process, labeled (a)-(d).

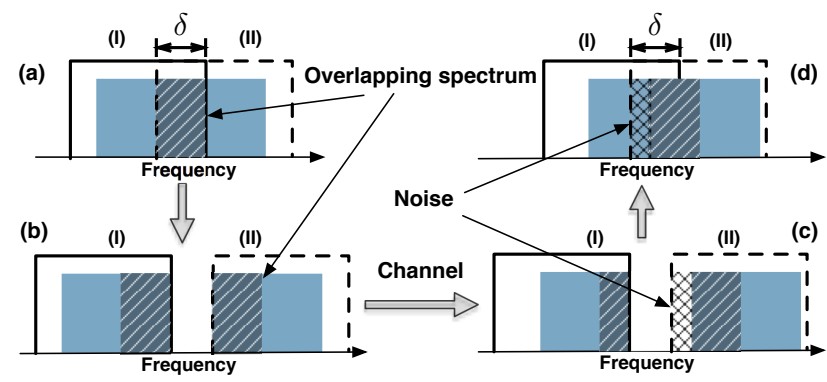

Figure 6: Spectrum shaping using two partially-overlapping filters. (a) Two subbands share an overlapping band $\delta$. (b) After postfilter modulation, each subband contains a copy of the overlapping spectrum $\delta$. (c) As a result of frequency drift at the receiver, only a portion of one subband is recovered while the other subband is recovered along with a noise band. (d) The overlapping spectrum $\delta$ ensures that the original spectrum can be reconstructed even if one subband is not recovered completely.

$$
\begin{array}{r}
X(f)=\hat{Y}(f), Y(f)=\hat{X}(f), \\
m_{1}^{(a)}(t)=1 / m_{1}^{(c)}(t), m_{2}^{(a)}(t)=1 / m_{1}^{(c)}(t), \\
m_{1}^{(c)}(t)=1 / m_{1}^{(a)}(t), m_{2}^{(c)}(t)=1 / m_{1}^{(a)}(t)
\end{array}
$$

where $\hat{Y}(f)$ is the spectrum of the received frame and $\hat{X}(f)$ is the spectrum of the reconstructed frame.

\subsection{Filter Design for Spectrum Shaping}

Prior work in spectrum shaping has largely adopted an OFDMbased approach $[25,30,32]$. While this approach draws upon many readily understood concepts similar to typical OFDM(A) modulation schemes, it has two significant disadvantages when applied to real-time spectrum shaping: (a) high overhead and complexity involved in maintaining strict time and frequency synchronization with pilot subcarriers, and (b) reduction in throughput due to the necessary use of a cyclic prefix to guard against inter-symbol interference.

RODIN mitigates these disadvantages with partially-overlapping finite-impulse response (FIR) spectrum shaping filters. Note that these FIR filters are only used for spectrum shaping. RODIN can support both OFDM and non-OFDM protocols using these FIR filters. RODIN itself is tolerant of timing drifts as time synchronization is handled by the attached COTS device as part of its PHY protocol; as long as the filtered spectrum encompasses the received frame, the COTS device can determine the appropriate 
frame boundary. RODIN is also resilient to frequency drifts by transmitting redundant spectral information through the use of partiallyoverlapping filters.

To understand this, consider the use of partially-overlapping filters to shape an input frame, as illustrated in Fig. 6. The two filters divide the spectrum into two portions, (I) and (II), that share a common overlapping subband of bandwidth $\delta$, as shown in Figs. 6(a) and (b). A frequency shift at the receiver, as shown in Fig. 6(c), causes some spectrum to be lost from (I) and noise to be introduced into (II). Observe that when the two subbands are recombined, the spectral information missing from (I) can be recovered from its redundant copy in (II). The degree of resilience to frequency drift is governed by the overlapping bandwidth $\delta$, which is a configuration parameter. We must ensure that the value chosen for $\delta$ is greater than the expected frequency drift. The lower bound on the overlapping bandwidth thus depends on the quality of the COTS device that RODIN is connected to. The effect of this noise is minimal since it is located at the very edge of the shaping filter and thus will be more heavily attenuated. Furthermore, this noise subband is typically very narrow as real-world measurements of actual frequency drift are shown to be small [4].

The overlapping bandwidth is also lower bounded by the amount of resources available on the FPGA: longer filters, which allow smaller overlapping bandwidths, require larger numbers of FPGA slices. The WARP platform used for our RODIN prototype can support a 64-tap filter.

The ideal requirements for a spectrum shaping filter are: (a) constant unit amplitude response and linear phase response in the passband, (b) narrow transition bandwidth, and (c) very high attenuation in the stopband. Unfortunately, neither the typical windowedapproach nor the Parks-McClellan algorithm can produce a filter that satisfactorily meets these three constraints. Thus, we adopt a constrained least squares algorithm [21] for filter design. We design our filters, using this algorithm, to have 64 taps, a passband ripple of $0.1 \mathrm{~dB}$ and an overlapping spectrum bandwidth that is approximately $10 \%$ of the total filter bandwidth.

\subsection{Spectrum-Shaping Latency}

We have implemented the spectrum shaper using a 64-tap FIR filter on the FPGA of the WARP platform to both validate its functionality and study the latency incurred in real-time spectrum shaping. The FPGA on the WARP runs at 40MHz.

The modulation and spectral combination steps consists of timedomain multiplication and addition, respectively. Each step thus incurs a latency of 1 clock cycle. The filtering step consists of a 64-tap time-domain convolution, and incurs a latency of 64 cycles. Note that the filtering latency is independent of the number of subbands used since all filters run in parallel on the FPGA.

The total latency of real-time spectrum shaping is therefore $64+$ $1+1=66$ cycles, or $1.65 \mu \mathrm{s}$ when running on the $40 \mathrm{MHz}$ FPGA. This spectrum-shaping latency is a mere $0.7 \%$ of the transmission time of a $1.5 \mathrm{~KB} 802.11 \mathrm{n}$ frame sent at $54 \mathrm{Mbps}$ (Rodin currently only supports SISO). Hence, a real-time spectrum shaping extension to commodity wireless hardware is feasible.

\section{PREAMBLE FOR SPECTRUM AGREE- MENT}

RODIN uses a unique preamble that is designed to indicate both the start of a frame as well as the spectrum bands it occupies.

\subsection{Challenges to Spectrum Agreement}

A frame sent by the transmitter can be decoded if and only if the spectrum occupied by the frame is known by the receiver. If the spectrum occupancy of a frame is unknown, the receiver can attempt to search for the frame over all the subbands. Assuming that a frame is known to occupy $M$ out of $N$ subbands, the receiver has to attempt to search for the frame over $N ! /(M !(N-M) !)$ possible subband combinations; if the bandwidth of the frame is unknown, this search space increases to $\sum_{m=1}^{M} N ! /(m !(N-m) !)$ subband combinations.

One might think of applying energy sensing to the subbands and decoding a frame using only the subbands with signal energy above a given threshold. This method, though simple, suffers from two serious limitations: (a) frequency-selective fading on the subband may result in a missed detection, and (b) in the case of multiple concurrent transmissions, each using a different set of subbands, it is impossible for a receiver to correctly map each occupied subband to its transmitter based on energy detection alone.

\subsection{I-FOP Design}

RODIN addresses this predicament by prepending a multi-subband preamble, I-FOP (In-Front Of Preamble), to the transmitted COTS frame. A unique preamble is assigned to each flow within the network, where a flow is simply a group of consecutive frames sent by the COTS device. This preamble must therefore be designed to (a) assign an address to each unique flow within the network, (b) specify the subband occupancy of each transmitted frame, and (c) enable the receiver to recover both the address and subband occupancy information of each frame without prior coordination with the transmitter. We stress that the spectrum occupancy can change from frame to frame even within the same flow.

A key feature that the preamble must possess is a strong correlation property - a receiver searching for a preamble $P$ via correlations must encounter a large correlation peak if and only if $P$ is present on the channel. Furthermore, this auto-correlation property must hold for a large set of sequences of the same length. This allows a different preamble to be assigned to each flow within a collision domain.

Zadoff-Chu (ZC) sequences [7] meet our requirements and are thus used in I-FOP. The length- $L$ discrete $\mathrm{ZC}$ sequence is defined as:

$$
x_{u}[n]=\exp \left(-j \frac{\pi u n(n+1)}{L}\right)
$$

where $u$ is the sequence ID and $0 \leq n, u \leq L-1$. ZC sequences have strong correlation properties that make them ideal for I-FOP: (a) the auto-correlation of a length- $L \mathrm{ZC}$ sequence with a cyclically-shifted version of itself is zero if $L$ is prime; (b) the cross correlation between two prime length ZC sequences is $1 / \sqrt{L}$.

RODIN selects a set $\left\{p_{0}, \ldots, p_{N_{F}-1}\right\}$ of ZC sequences to address a flow. The bandwidth of each frame within the flow occupies $N_{F}$ subbands. RODIN applies a random cyclic shift to each sequence before constructing the preamble for the flow. The crosscorrelation property reduces the chance of collision in the event that the same ZC sequence is selected by multiple transmitters. With this approach, there is a large set of $L^{2} \mathrm{ZC}$ sequences of length- $L$ that can be used to construct preambles.

Let $\mathbf{f}=\left\{f_{0}, \ldots, f_{N_{F}-1}\right\}$ be the set of $N_{F}$ subbands that RoDIN uses to transmit a frame. The preamble constructed for this particular frame is specified by the set $\mathbf{S}=\left\{S_{f_{k}}^{p_{k}}: 0 \leq k \leq N_{F}-1\right\}$, where $S_{f_{k}}^{p_{k}}$ indicates that sequence $p_{k}$ is transmitted on the subband $f_{k}$ and $f_{0} \leq \ldots \leq f_{N_{F}-1}$. The time-domain representation of the preamble is:

$$
y[n]=\sum_{k=0}^{N_{F}-1} x_{p_{k}}[n] \cdot e^{-j 2 \pi f_{k} n / N}
$$


for $0 \leq n \leq L-1$

\subsection{I-FOP Detection}

We assume, for now, that the transmitter and the receiver know the set of $\mathrm{ZC}$ sequences, $\left\{p_{0}, \ldots, p_{N_{F}-1}\right\}$, used to address the flow between them. The receiver faces the challenge of determining the set of subbands $\left\{f_{0}, \ldots, f_{N_{F}-1}\right\}$ occupied by the transmitted frame.

Let $\hat{\mathbf{S}}=\left\{\hat{S}_{f_{k}}^{p_{k}}: 0 \leq k \leq N_{F}-1\right\}$ be the preamble that is detected by the receiver. This preamble detection procedure uses the following two properties of the transmitted preamble.

(a) The known order of the sequences. Given the set of $\mathrm{ZC}$ sequences, $\left\{p_{0}, \ldots, p_{N_{F}-1}\right\}$, used in the preamble, $\hat{\mathbf{S}}$ must be found such that $f_{0}<f_{1}<\ldots<f_{N_{F}-1}$. This increases the number of possible preambles by allowing for different preambles to be constructed using the same set of ZC sequences, but with different subband orders.

(b) Location of the correlation peaks. Multiple ZC sequences sent by the same transmitter as part of a single preamble will arrive at the receiver at approximately the same time. However, due to frequency-selective fading, the peaks may not be precisely aligned in time. To account for this, we use a threshold, $\xi$, to limit the range of acceptable separation between peaks - only sets of correlation peaks that are within $\xi$ samples apart are considered as candidates for the preamble.

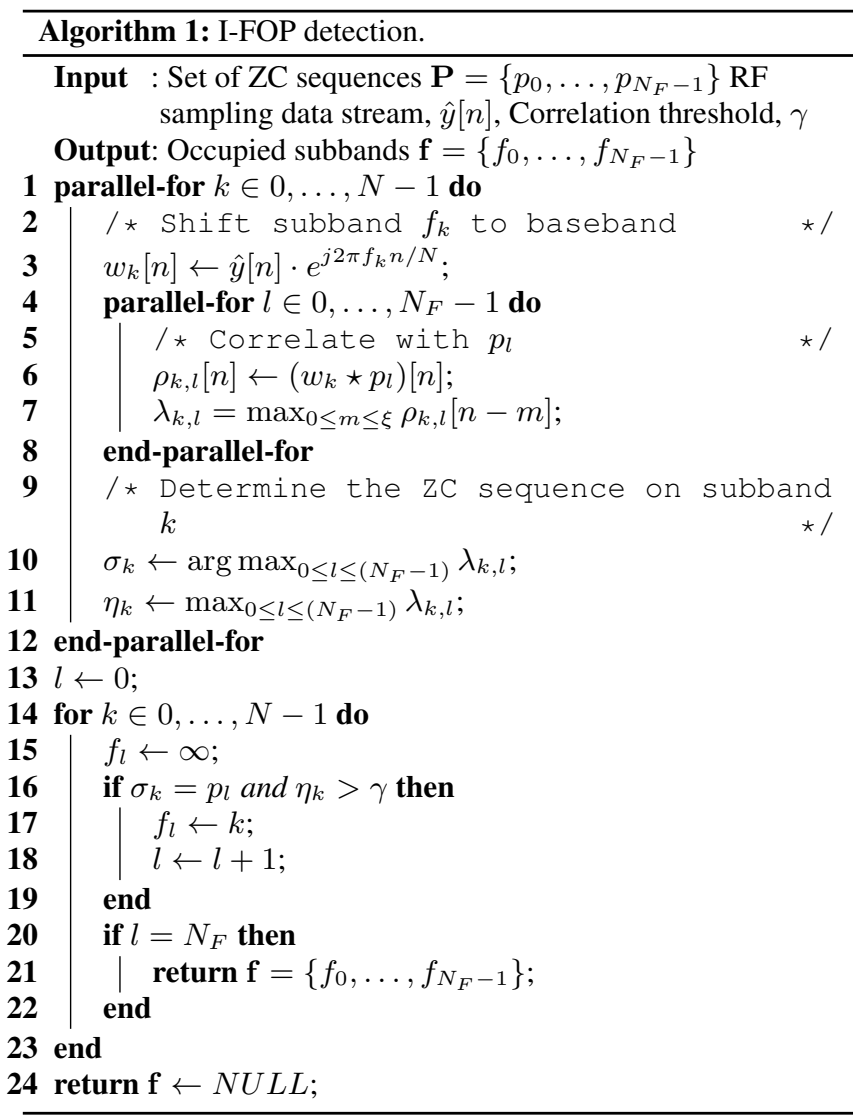

Algorithm 1 shows the pseudocode of the multi-preamble detection. In lines 1-12, RODIN searches for the ZC sequence that is transmitted in each subband. Observe that we use parallelfor loops for this search step since in an FPGA implementation, all iterations of these parallel-for loops can be executed concur-
Table 1: Time required for preambles constructed with $\mathrm{ZC}$ of length 37,73 and 113 to be transmitted at 5,10 and $20 \mathrm{MHz}$ bandwidths.

\begin{tabular}{|c|c||c|c|c|}
\cline { 3 - 5 } \multicolumn{2}{c|}{} & \multicolumn{4}{c|}{ Preamble Length } \\
\cline { 3 - 5 } \multicolumn{2}{c|}{} & 37 & 73 & 113 \\
\hline \multirow{3}{*}{ BW $(\mathrm{MHz})$} & 5 & $7.4 \mu \mathrm{s}$ & $14.6 \mu \mathrm{s}$ & $22.6 \mu \mathrm{s}$ \\
\cline { 2 - 5 } & 10 & $3.7 \mu \mathrm{s}$ & $7.3 \mu \mathrm{s}$ & $11.3 \mu \mathrm{s}$ \\
\cline { 2 - 5 } & 20 & $1.8 u s$ & $3.65 \mu \mathrm{s}$ & $5.56 \mu \mathrm{s}$ \\
\hline
\end{tabular}

rently to reduce the search time. In lines 13-24, RoDIN searches for a set of subbands $\left\{f_{0}, \ldots, f_{N_{F}-1}\right\}$ that contain the sequences $\left\{p_{0}, \ldots, p_{N_{F}-1}\right\}$ such that $f_{0}<\ldots<f_{N_{F}-1}$ must hold. Note that this for loop cannot be parallelized since the result of each iteration depends on the result of the previous iteration.

\subsection{Inter-Subband Interference}

Observe that RoDIN does not apply any filter to isolate each subband before conducting a search for a $\mathrm{ZC}$ sequence. This choice is made to avoid the additional delay that comes with a filtering step. However, there is now a possibility that sequences on different subbands interfere with each other during the correlation-based search. This possibility is present regardless of the type of sequence used, e.g., Gold, ZC, Walsh-Hadamard, etc. However, we argue that the possibility of inter-subband collisions in our preamble design is very low.

A collision between two subbands can occur only if two or more different transmitters (a) select the same ZC sequence, (b) apply the same cyclic shift to the sequence, and (c) transmit at almost the same time. We posit that the probability of all three events occurring at even two non-colluding transmitters is very low. To gain some insight into this, first recall that in CSMA networks, the random backoff process undertaken by each transmitter minimizes the possibility of simultaneous transmissions. Even if simultaneous transmissions do occur, the set of $\mathrm{ZC}$ sequences can be made large enough to minimize the probability of collisions. For example, if we use ZC sequences of length 73 , there are a total of $73 \times 73=5329$ possible sequences that can be used by RoDIN. The probability of two devices picking the same sequence is a mere $(1 / 5329)^{2}=3.5 \times 10^{-8}$. Hence, inter-subband interference does not affect the performance of I-FOP.

\subsection{I-FOP Delay}

The spectrum-shaping delay incurred by I-FOP depends on two parameters: the length of the chosen $\mathrm{ZC}$ sequence, and the bandwidth at which each sequence is transmitted. Table 1 shows the transmission time required for each sequence built from $\mathrm{ZC}$ codes of 37,73 and 113 samples long at 5, 10 and $20 \mathrm{MHz}$. These subband bandwidths are suitable for use by 802.11 devices. The bandwidth of each transmitted sequence $S_{f_{k}}^{p_{k}}$ must be no larger than the bandwidth of each subband.

The delay at the receiver is due mainly to the processing time needed to find I-FOP. For every new sample, $\hat{y}[n]$, received by the detector in Algorithm 1, the parallel-for loops operate in constant $O(1)$ time while the search in lines 13-24 takes $O(N)$ time. With sufficient FPGA resources for full parallelism, the search can be completed in $N$ clock cycles, or $(0.0225 N) \mu s$ with a $40 \mathrm{MHz}$ FPGA.

As an example, if we spectrally shape a $20 \mathrm{MHz} 802.11 \mathrm{n}$ over a $B=40 \mathrm{MHz}$ RF bandwidth using the 64-tap filter from $\$ 3.3$ and a preamble based on a length-37 ZC sequence, the overall delay is $1.65+7.4=9.05 \mu \mathrm{s}$. This is merely $3.8 \%$ of the transmission 
time of a 54Mbps $802.11 \mathrm{n}$ frame. The delay incurred by I-FOP may exceed the SIFS delay of WiFi COTS devices and trigger an ACK timeout at the transmitter. However, these ACK timeouts can be easily changed in software [1] and do not pose a hurdle to SDRCOTS integration. This local SIFS modification allows the attached COTS device to account for the extra delay from I-FOP ; other nonRODIN WiFi devices can operate normally without modifications.

\subsection{Preamble Address Assignment}

RODIN devices must assign an address to each flow in a distributed manner before spectrum agreement between devices is completed. Addresses to new flows are assigned using an association frame.

An association frame is a control frame sent between RoDIN devices, and is not passed to the COTS device. Each association frame is spectrally shaped to occupy only the available subbands and is prepended with a preamble constructed using a fixed set of $\mathrm{ZC}$ sequences. This set of ZC sequences is the association set and is known to all RoDIN devices. The association frame contains only the IDs of the ZC sequences and the order in which they will be used.

A RODIN receiver searches all subbands for the association set. Once this association set is found, RODIN recovers the association frame using the spectrum shaper from $\S 3$. It then decodes the frame to obtain the $\mathrm{ZC}$ sequence information that will be used for subsequent frames from the same flow. Once an address has been assigned, all transmissions belonging to the same flow, even if they originate from different RODIN devices (e.g., DATA and ACK frames), use the same preamble address.

Since the information carried in the association frame is small, the size of the frame is small, especially when compared with the total size of the flow. Hence, the overhead of address assignment is negligible.

\subsection{Subband Selection}

The transmitter selects the subbands by choosing the $N_{F}$ subbands that have the lowest energy levels at the point of frame transmission. We make use of an FFT (Fast Fourier Transform)-based energy detector - we take the FFT of incoming samples and measure the magnitude of the energy in each subcarrier. On the $40 \mathrm{MHz}$ FPGA, for example, a 128-bin FFT takes approximately $5 \mu \mathrm{s}$. Hence, energy values at any point in time are delayed by about $5 \mu \mathrm{s}$. This is acceptable since the channel state does not vary significantly over that short duration. Note that energy sensing delay decreases as the FFT length gets shorter.

On a faster and larger FPGA, we can also implement more advanced spectrum-scanning techniques, such as those based on the Spectrum Correlation Function [13]. This will enable RoDIN to not only detect the currently occupied subbands, but also determine the protocol occupying them and predict future usage patterns of the interferer.

\section{SPECTRUM MANAGEMENT}

Algorithm 2 shows the pseudocode that defines the operation of the Spectrum Manager. RODIN is in the receive state until frames are detected from the COTS device. In this state, the RX spectrumshaping filters are configured to span the occupied spectrum indicated by each received I-FOP.

When a frame is transmitted by the COTS device, RODIN first configures the TX spectrum-shaping filters and TX I-FOP to span the transmit spectrum subbands. The preamble is then transmitted while the samples from the COTS device are filtered and modu-
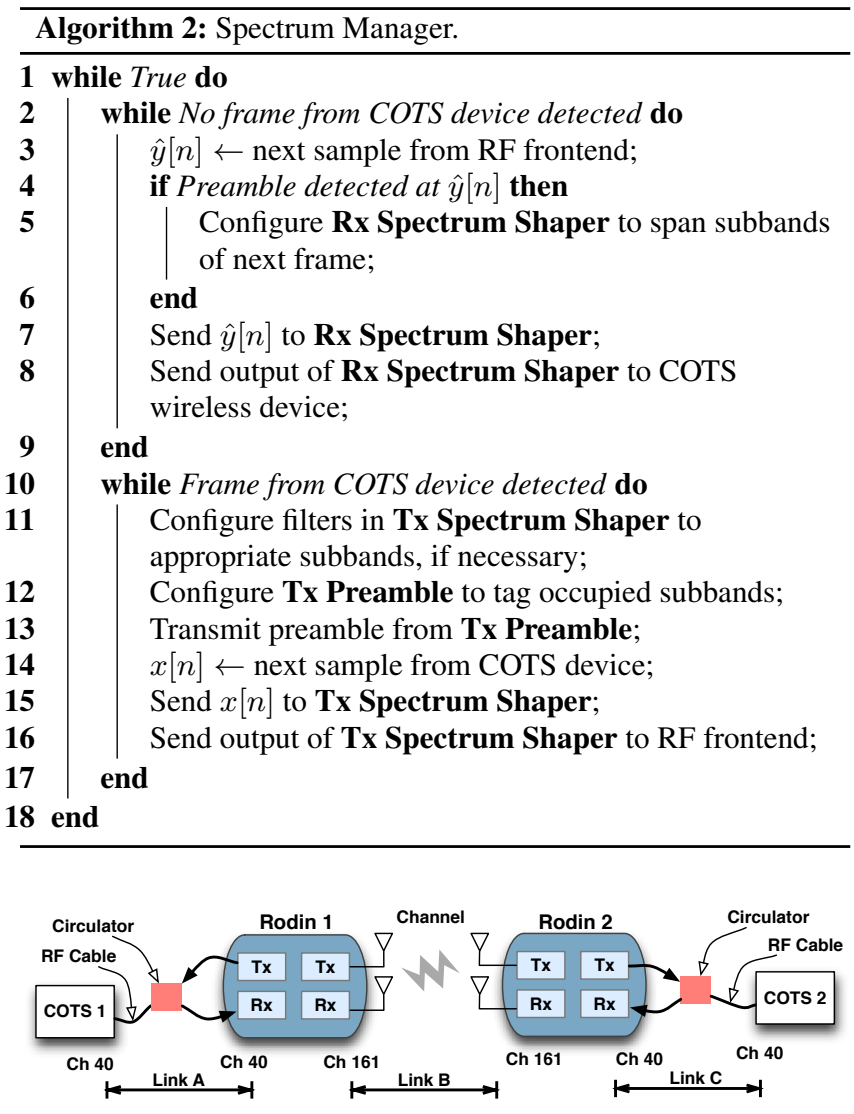

Figure 7: Experimental setup. Each RoDIN device is connected to a COTS device via a coaxial cable.

lated. The spectrally shaped samples are transmitted after I-FOP transmission is complete.

\section{EVALUATION: SPECTRUM SHAPING}

\subsection{Experiment Setup}

Fig. 7 illustrates the setup used for evaluating the performance of individual RODIN devices. Each RODIN spectrum shaper is implemented in Verilog/VHDL and runs on the FPGA of a WARP platform with four radios. Each radio is permanently set to either the Tx or Rx mode. One pair of Tx/Rx radios from each WARP device is connected to a circulator that is then connected to a COTS device. These connections are made using coaxial cables. A circulator routes passband signals between the COTS device and the two radios on the WARP - analog signals coming from the COTS device is sent only to the Rx radio on the WARP, while signals from the Tx radio on the WARP is routed only to the COTS device. Signals between the Rx and Tx radios are blocked by the circulator.

The circulator is used here so that RODIN can receive frames from the COTS device without the Tx-Rx switching delay that will otherwise be incurred by the radio hardware if only one radio is connected to the COTS device. The other two Tx/Rx radios on each WARP device are connected directly to antennae. The two RoDIN devices are placed approximately $2 \mathrm{~m}$ apart. We have successfully used Ralink 802.11a WiFi card for COTS 1 and 2. However, to achieve finer-grained control of the transmitted signal for experi- 

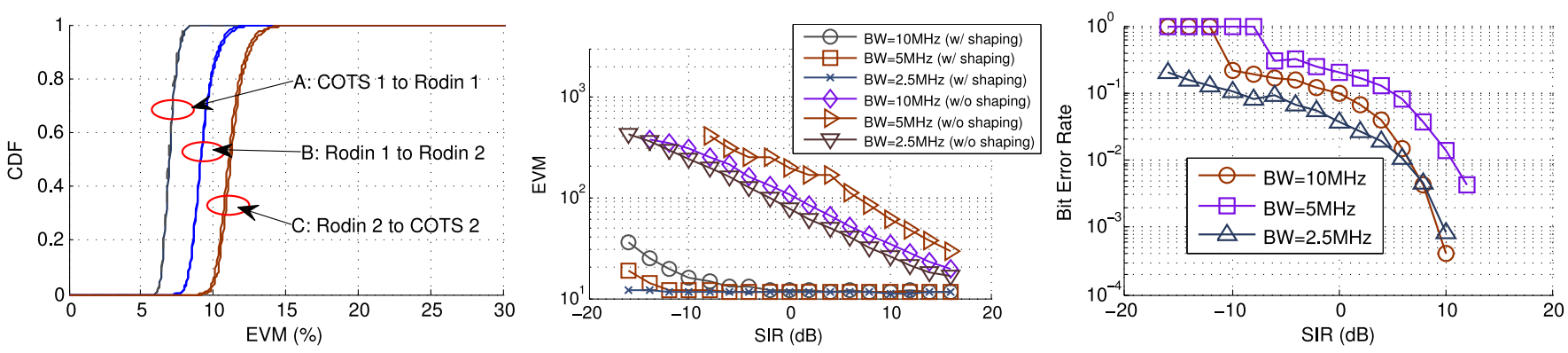

Figure 8: EVM of symbols in an OFDM Figure 9: Mean EVM of OFDM frames mea- Figure 10: BER of OFDM frames measured frame with and without spectrum shaping. No sured at COTS 2 under different SIR levels. interference.

at COTS 2 without shaping. No errors are encountered when spectrum shaping is used.

mental purposes, we use WARP for COTS 1 and 2 for the rest of the experiments.

We send uncoded OFDM frames with a bandwidth of $10 \mathrm{MHz}$ between the two COTS devices. The spectrum of the OFDM frames can be shaped to span any $10 \mathrm{MHz}$ of spectrum within the $20 \mathrm{MHz}$ maximum bandwidth supported by each radio. For all experiments in this section, we split the 10MHz OFDM frame into two subbands of $5 \mathrm{MHz}$ each. These subbands are transmitted with a $10 \mathrm{MHz}$ separation between them.

Each RODIN device detects transmissions from its attached COTS device by checking the RSSI of the Rx radio that is directly connected to the circulator. If the RSSI exceeds a predefined threshold, the COTS device is assumed to be transmitting. This can be done easily as the SNR of transmissions over the coaxial cable is high. At all other times, the Tx radio continuously transmits received signals to the COTS device for receiver processing. This maintains the capability of the COTS device to overhear transmissions from other devices that share the same discontiguous spectrum.

We use two metrics to measure the performance of the spectrum shaper: Error Vector Magnitude (EVM), which is shown as a percentage, and Bit Error Rate (BER), which is the fraction of bits received in error.

\subsection{Spectrum Shaping Results}

Without Interference. We transmit 2,000 OFDM frames using QPSK symbols from COTS 1 to COTS 2 using the setup in Fig. 7, and measure the mean EVM of the frames between each pair of directly connected devices. This experiment is conducted twice, once with and once without spectrum shaping. Fig. 8 shows the CDF of measured EVM. One important conclusion from this result is: Spectrum shaping does not distort the signal. The CDF of the EVM over each OFDM frame is identical with and without spectrum shaping of the transmitted OFDM frame. Hence, real-time spectrum shaping can be implemented in the FPGA without any loss of signal quality.

Direct manipulation of a signal from a COTS device with an attached RODIN platform does introduce some distortion into the signal. The median EVM of frames sent over Link $A$ of Fig. 7 is $7 \%$ while median EVM of the frame that is spectrally shaped and sent over Link $B$ is $9 \%$. Finally, the transmission over Link $C$ to COTS 2 increases the median EVM to $11 \%$. (An EVM of $11 \%$ is small enough not to increase BER; BER of all frames transmitted in Fig. 8 is zero.) These additional distortions are introduced during (a) up and down signal modulation by the AD/DA converters at both COTS devices and the radios on the WARP, and (b) time and frequency offsets between the COTS device and its attached WARP. Both of these sources of distortion can be eliminated by tighter in- tegration between RODIN and the COTS device: distortion due to up/down converters can be reduced by passing the baseband signal directly between RODIN and the COTS device; distortion due to time and frequency offsets can be mitigated by synchronizing RODIN with the clock used by the COTS device.

With Interference. We transmit an interfering signal using another WARP device. The transmission power of this signal is varied to achieve a range of Signal-to-Interference Ratios (SIR). At each interference power level, we transmit the interference at three different bandwidths-2.5, 5 and $10 \mathrm{MHz}$. Fig. 9 shows the EVM of a $10 \mathrm{MHz}$ OFDM frame sent from COTS 1 to COTS 2 that experiences interference with bandwidth $2.5,5$ and $10 \mathrm{MHz}$. This experiment is conducted over a range of SIR levels, with and without RODIN spectrum shaping.

We first consider the performance of spectrum shaping. The mean EVM of the OFDM transmission when SIR is greater than $-2 \mathrm{~dB}$ is $11 \%$. This is equivalent to a spectrum-shaped OFDM transmission in the absence of interference, as shown in Fig. 8. At SIR levels lower than $-2 \mathrm{~dB}$, the impact of interference on the OFDM transmission depends heavily on the interference bandwidth - interference with a $10 \mathrm{MHz}$ bandwidth increases the EVM to almost $40 \%$ while it remains at $11 \%$ when the bandwidth is $2.5 \mathrm{MHz}$. This variation is due to the fact that filters used to generate the interference signal are not ideal. Hence, some energy leakage occurs at the edges of the filter. Although the two subbands of the spectrumshaped OFDM frame are separated by $10 \mathrm{MHz}$, they are still affected by the leaked interference energy. With a $10 \mathrm{MHz}$ interference bandwidth, the leakage energy is sufficient to distort the spectrum-shaped transmission. At $2.5 \mathrm{MHz}$, the bandwidth of the interference is small enough that power leakage due to imperfect filters does not have a noticeable impact on the main OFDM transmission.

Without spectrum shaping, the narrowband interference has a significant impact on the OFDM transmission. For a given interference power, the smaller the interference bandwidth, the greater the interference power per subcarrier. The effect of this is seen from the fact that the distortion of the OFDM frames from the $5 \mathrm{MHz}$ interference is greater than that from the $10 \mathrm{MHz}$ frames-the increased interference power on fewer subcarriers is high enough to make up for the reduction in the number of subcarriers that encounter interference. When the interference bandwidth is at $2.5 \mathrm{MHz}$, the small number of subcarriers affected allows the EVM to fall below that when a $10 \mathrm{MHz}$ interference is used.

This behavior is also evident when we consider the BER of the OFDM frames, as shown in Fig. 10. With spectrum shaping, the primary OFDM frames are sent on frequency bands that are not occupied by the interfering signal. The BER is thus zero for spectrum- 

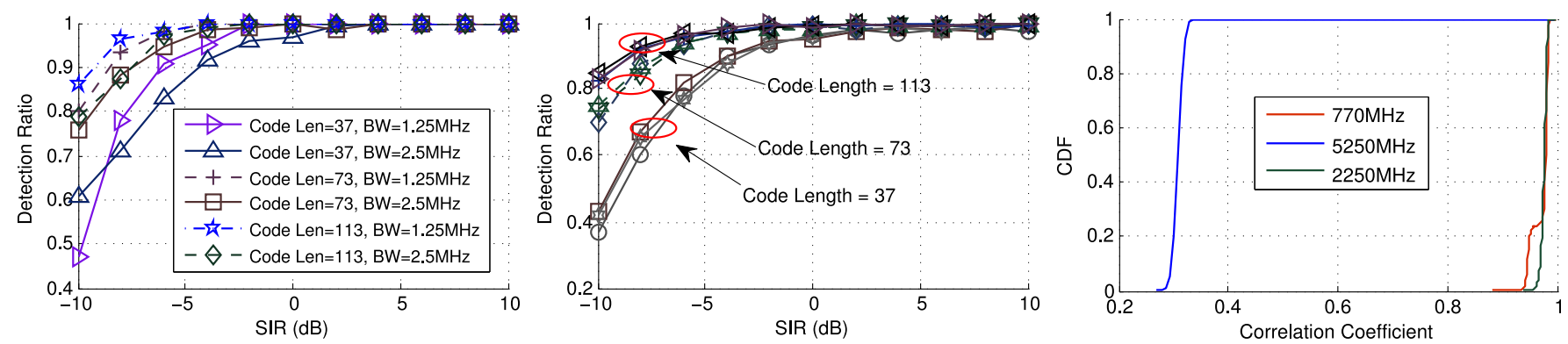

Figure 11: Preamble detection rate of three Figure 12: Preamble detection rate of three Figure 13: CDF of the correlation of the RSSI codeword lengths over $N=8$ subbands on different codeword lengths over $N=8$ sub- seen across all measurement slots over time. a $20 \mathrm{MHz}$ channel in the presence of interfer- bands on a $20 \mathrm{MHz}$ channel. Each preamble is ing preambles. Each preamble is transmitted transmitted under 0, 12 and 20dB SNR.

at $2.5 \mathrm{MHz}$ and $1.25 \mathrm{MHz}$.

shaped OFDM frames. Without spectrum shaping, the OFDM frame has a BER of 1.0 when it encounters a 10 or $5 \mathrm{MHz}$ interference at SIR below $-12 \mathrm{~dB}$. The BER of the OFDM frame with a $2.5 \mathrm{MHz}$ interference is expectedly lower than that at interference bandwidths of 5 and $10 \mathrm{MHz}$, but still stands at a high $1 \%$ at $8 \mathrm{~dB}$ SIR.

\section{EVALUATION: I-FOP}

\subsection{Experiment Setup}

We evaluate I-FOP using five WARP devices placed at various locations around an office. Since the objective of this experiment is to evaluate the feasibility and performance of our preamble design, we run experiments using only WARPLab+MATLAB instead of an FPGA-based WARP implementation. The results obtained using WARPLab and an FPGA implementation will be identical.

The performance of I-FOP is evaluated under SIRs ranging from -10 to $10 \mathrm{~dB}$. This interference consists of different I-FOP that overlaps with the transmission of the primary I-FOP. The result for each SIR is the mean of 2,000 preamble transmissions. In each transmission, we select a random receiver, transmitter and interferer from five WARP devices. We use a $20 \mathrm{MHz}$ channel with $N=8$ subbands (each subband is thus $2.5 \mathrm{MHz}$ wide). Three different preamble lengths are evaluated: 37, 73 and 113 samples. For every preamble, we randomly select $N_{F}=4$ subbands and transmit a different $\mathrm{ZC}$ sequence on each one. All ZC sequences are transmitted at the same bandwidth.

The receiver searches for the known $\mathrm{ZC}$ sequences that belong to the primary preamble transmission using the procedure shown in Algorithm 1. If the set of ZC sequences is found in the specified order, the preamble is considered to be detected. Otherwise, a missed-detection is recorded.

We also evaluate the performance of the preamble under varying SNR levels. However, due to the difficulty of accurately controlling the noise level in the channel, SNR evaluations are conducted using a simulated 802.11 channel.

\subsection{Experimental Results}

Fig. 11 shows the detection probability of preambles with 3 different lengths, in the presence of overlapping interfering preambles. We run two experiments, with each one conducted over a range of SIR values. In the first experiment, each ZC sequence of every preamble (both the intended and interfering preambles) is sent at $2.5 \mathrm{MHz}$ (equal to the bandwidth of the subband); in the second experiment, each $\mathrm{ZC}$ sequence is sent at $1.25 \mathrm{MHz}$, half the subband bandwidth. Interfering preambles are transmitted with a random time offset with respect to the non-interfering ones.

Under varying SIR levels. Observe that for preambles with the same length, the detection accuracy is greater as the bandwidth of each ZC sequence is reduced for two reasons. First, as the sampling rate of WARP is constant, the longer correlation period that results from a lower bandwidth $\mathrm{ZC}$ sequence gives a higher correlation peak magnitude when a match is found. Second, when ZC sequences are transmitted at $1.25 \mathrm{MHz}$, there is a guard band between sequences on adjacent subbands. This reduces the inter-subband interference that arises due to energy leakage from adjacent subbands. No guard bands are present when the $\mathrm{ZC}$ sequences are sent at $2.5 \mathrm{MHz}$.

Also, observe that the detection ratio increases with increasing $\mathrm{ZC}$ sequence length. This is because the peak auto-correlation magnitude is proportional to the sequence length $L$, while the crosscorrelation magnitude of $1 / \sqrt{L}$ actually decreases with increasing sequence length. These two effects cause the SNR of the correlation peak to increase with increasing $\mathrm{ZC}$ sequence length.

Under varying SNR levels. The accuracy of the preamble detector is similar over a wide range of SNR values, as shown in Fig. 12. For each ZC sequence length, we transmit the preamble at 0,12 and $20 \mathrm{~dB}$ SNR. Observe that accuracy is largely unaffected by the SNR level on the channel and is primarily dependent on the interference power.

In our experiments, the probability of detecting an I-FOP preamble when no I-FOP is present (false positive) is zero. False positives may occur due to ZC sequence collisions or more complicated channel fading scenarios. We can mitigate the effects of fading by using Rake correlators to search for the ZC sequences. However, false positives have limited impact on the operation of RODIN as the falsely received frame/signal are simply discarded by the COTS device.

\section{EVALUATION: RODIN}

We evaluate the performance of RODIN using simulations over detailed channel measurements from [29]. These channel measurements show the usage behavior of devices that operate on three separate bands. During periods when the channel RSSI is low, primary user activity is absent and spectrum agile devices can transmit opportunistically. Our objective is to show the efficacy of per-frame spectrum shaping in using these short-term transmission opportunities.

\subsection{Simulation Setup}

Trace data. Each channel measurement of [29] spans a $1.6 \mathrm{GHz}$ 
bandwidth that is centered at three different frequencies 770, 2250 and $5250 \mathrm{MHz}$, so they cover the $2.4 \mathrm{GHz}$ and $5 \mathrm{GHz}$ ISM bands used by WiFi devices. Measurements were taken over several days at three different locations: for brevity, we only show results using the data set measured at rooftop of a school. Each sweep over the entire $1.6 \mathrm{GHz}$ bandwidth takes about $1.8 \mathrm{~s}$ and captures 8,192 samples, with each sample spanning $200 \mathrm{kHz}$. Although the measurement data does not capture channel usage patterns shorter than $1.8 \mathrm{~s}$, channel statistics have been shown to remain unchanged at shorter time scales [15]. This strongly suggests that we can expect such statistics to be present at sufficiently small time scales to make RODIN useful. Hence, our analysis using this data is still applicable even when considering finer-grained channel usage patterns.

Device models. We model three different types of wireless devices in our simulations; two that support spectrum shaping and one that does not. The maximum RF bandwidth of each device is $20 \mathrm{MHz}$. The bandwidth of transmitted signal is $10 \mathrm{MHz}$, with the remaining $10 \mathrm{MHz}$ bandwidth used for spectrum reallocation. There are three models as follows.

(1) RodIN. This model uses per-frame spectrum shaping and the multi-subband preamble. At the beginning of each measurement slot (1.8s), RODIN measures the RSSI of all subbands and selects the $N_{F}$ subbands with the lowest RSSI. This is equivalent to selecting the set of $N_{F}$ subbands with the lowest interference powers. If all subbands have RSSIs lower than a predefined threshold, RODIN transmits a frame over those time slots. RODIN can carry out this measure-shape-transmit process within a single time slot due to its per-frame spectrum agreement and shaping capability. The performance of RODIN is modeled based on the I-FOP detection probability measured in the previous section.

(2) COTS-Spec. This model can bond multiple subbands for a single transmission, but cannot change the bonding on a per-frame basis. At the beginning of a time slot (1.8s), it selects the $N_{F}$ subbands with the lowest RSSI as before. However, these selected subbands are used only in the next time slot. The set of subbands used for the current transmission is selected in the previous time slot. This represents the delay required by a COTS device to switch to a different set of subbands. Note that this is an optimistic model because (a) we do not consider the additional overhead required for spectrum agreement and (b) we assume that COTS-Spec can continue to transmit in the current time slot even as it is changing its set of bonded subbands.

(3) COTS-Mono. In this model, the COTS device makes use of the middle $10 \mathrm{MHz}$ bandwidth for transmitting a frame, but no spectrum shaping is used. This represents a typical 802.11-type device that uses monolithic spectrum blocks for transmission.

Channel model. We are interested in finding the number of time slots during which each of these models can find a transmission opportunity. To evaluate the performance of each model, we partition each of the three traces into $20 \mathrm{MHz}$ channels (for a total of 81 channels) and simulate the operation of each model on all the channels. Each channel is divided into $N$ subbands, of which $N_{F}$ is used by the frame transmission. We repeat the experiments for RODIN and COTS-Spec using different subband bandwidths, $b$, and vary $N$ and $N_{F}$ to maintain $N \cdot b=20 \mathrm{MHz}$ and $N_{F} \cdot b=10 \mathrm{MHz}$. The threshold levels that we use for 770,2250 and $5250 \mathrm{MHz}$ trace sets are $-100,-90$ and $-90 \mathrm{dBm}$, respectively. These are chosen to be similar to the 802.22 standard for $770 \mathrm{MHz}$ data set and the 802.11 standard for the others. We assume that there is only a single transmitter-receiver pair in each channel as it is sufficient to capture the behavior of the device models under a wide range of channel conditions. We leave the study of RODIN-to-RODIN interference to future work.

\subsection{Simulation Results}

Channel characteristics. The gain from per-frame spectrum shaping depends on the temporal variability-the more frequently the interference level on the channel changes, the greater the need for fast spectrum shaping. Fig. 13 shows the correlation coefficient of the RSSI on each measurement slot over time, for each trace set. Channels within the $5250 \mathrm{MHz}$ data set experience high temporal variability and have a median correlation coefficient of about 0.3 . On the other hand, channels within the 770 and $2250 \mathrm{MHz}$ data sets experience minimal temporal variability, as seen by the high correlation coefficients. We expect the gain from per-frame spectrum shaping to thus be greater in the $5250 \mathrm{MHz}$ channels than in channels at other frequencies.

Transmission time slots. Fig. 14 shows the proportion of time slots in each channel in which the different devices can find transmission opportunities. Note that the channels are labeled in increasing order of their center frequencies. In the $5250 \mathrm{MHz}$ trace set, as shown in Fig. 14a, the high temporal variability of the channel means that subbands found to be available for transmission in one time slot are unlikely to still be available in the next time slot. Hence, COTS-Spec with $1 \mathrm{MHz}$ subbands can only transmit in up to $15 \%$ time slots. COTS-Spec with $2 \mathrm{MHz}$ subbands fails to find any transmission slots. A surprising result is that the performance of COTS-Mono is almost identical to that of COTS-Spec with $1 \mathrm{MHz}$ subbands. This shows that under highly varying channels, slow channel adaptation with narrow subbands performs almost identically to no spectrum adaptation; while slow channel adaptation with wider subbands fails to find any transmission opportunities.

The per-frame spectrum shaping of RODIN enables it to transmit on a significantly larger proportion of the time slots-up until $95 \%$ of the time slots in channel 81 . Furthermore, we note that time slot utilization is increased when we use smaller subband bandwidthsRoDIN using $1 \mathrm{MHz}$ subbands $\left(N=20, N_{F}=10\right)$ can outperform the same device using $2 \mathrm{MHz}$ subbands $\left(N=10, N_{F}=5\right)$ by more than $50 \%$ in some channels. Note that channels $1-50$ in the $5250 \mathrm{MHz}$ data set fall into spectrum that is completely occupied by interferers. Hence, no slots can be found by any devices.

The performance of COTS-Spec improves under the low temporal variability of the 770 and $2250 \mathrm{MHz}$ trace sets. Fig. $14 \mathrm{~b}$ shows that the fraction of time slots used by COTS-Spec is almost equal to that used by RoDIN for transmissions. However, in Fig. 14c, we see that even in channels with high correlation coefficients, RODIN still finds more transmission opportunities than COTS-Spec at the same subband bandwidth. This is seen between channels 20 and 30 . COTS-Mono performs poorly even on channels with low temporal variation, as shown in both Figs. 14b and 14c. Spectrum shaping is still necessary here as the low temporal channel variability does not imply the widespread availability of high bandwidth channels.

\section{DISCUSSION}

SDR-COTS hybrid. Our experimental setup in Fig. 7 is an example of an SDR-COTS hybrid, which enables interesting experiments impossible by either of COTS or SDR only. The SDR handles only the necessary PHY-layer manipulations, while the COTS device handles the main MAC/PHY processing. Since we need not support a full MAC/PHY protocol on RoDIN, we use all the resources on the FPGA for a spectrum shaper and 64-tap spectrum correlation function. This hybrid architecture is applicable to both OFDM and non-OFDM-based COTS devices.

Interaction with COTS devices. Increasing the SDR-COTS integration can improve the performance of per-frame spectrum shaping. Using rate adaptation as an example, the SDR can provide the 


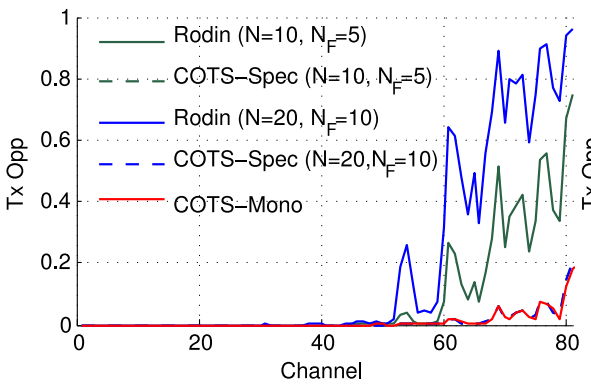

(a) $5250 \mathrm{MHz}$.

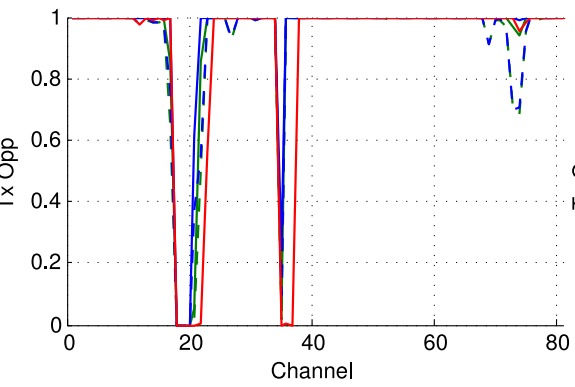

(b) $2250 \mathrm{MHz}$.

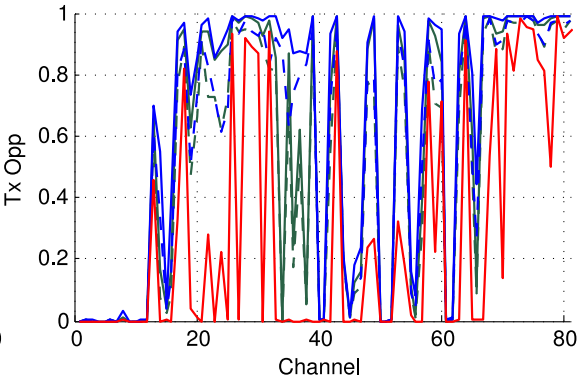

(c) $770 \mathrm{MHz}$.

Figure 14: Proportion of time slots that each of the devices, RODIN, COTS-Spec and COTS-Mono, can transmit in.

COTS hints on the SNR of other channels, so that the COTS device can immediately select the appropriate rate to match the per-frame spectrum when a spectrum reallocation is performed.

Per-frame spectrum shaping in the network. RODIN transparently combines multiple spectrum fragments into a contiguous virtual channel that is seen by the COTS device. Since it obtains these spectrum fragments with a CSMA policy, we expect multiple RoDIN nodes to interact without the need for more complex channel access protocols. Our current RoDIN prototype is limited to single-link operation and we leave more detailed network-scale studies to future work.

COTS devices using non-contiguous spectrum. RODIN is designed for the case where the RF bandwidth of the SDR frontend is larger than that of the COTS device. At present, RoDIN does not support COTS devices using non-contiguous bandwidths. As the SDR/ASIC platform evolves and supports larger bandwidths, RODIN can be extended to support non-adjacent frequency blocks. RODIN with more than two spectrum shaping filters. Our experimental evaluation of spectrum shaping uses only two shaping filters due to FPGA resource constraints. However, given a larger FPGA, we can increase the number of shaping filters in RODIN. Furthermore, this can be accomplished while keeping the total overlapping bandwidth unchanged.

RODIN with wideband COTS devices. The variability in the channel response is known to increase with channel bandwidth. Hence if RODIN spreads a wideband spectrum (such as a $80 \mathrm{MHz}$ signal from an 802.11ac device) to an even wider band, additional processing steps such as RODIN-specific pilots might be necessary to compensate for the greater distortion seen on the channel. Other parameters, such as the overlapping bandwidth of the filters, might also need to be adjusted. However, since wideband COTS devices will already have built-in capability to accommodate the greater channel distortions, the modifications needed for RODIN might be minimal.

\section{RELATED WORK}

Spectrum Agility. WhiteFi [3] is a variable-bandwidth 802.11based prototype that provides protocols that govern channel-switch triggers, channel probing and selection in whitespaces. This idea of variable-bandwidth communications is also used by FLUID [20] in enterprise networks. Jello [30] extends this variable bandwidth idea to support non-contiguous channel bonding in challenging networks. TIMO [10] adopts a different approach to handling interference on MIMO channels, treating interference as a single MIMO streams while simultaneously transmitting frames on the remaining MIMO streams. SVL [25] and Picasso [14] are both spectrum- shaping layers for general wireless devices. However, these solutions require tight integration with the COTS device's PHY and are not fast enough to support per-frame shaping. The new IEEE 802.11 ac standard draft also specifies non-contiguous $80+80 \mathrm{MHz}$ channel bonding as an optional feature [2], but does not support per-frame shaping. SWIFT [19] supports transmissions over noncontiguous bands while avoiding interference from narrowband devices. However, it differs from RODIN as it does not support perframe spectrum shaping and agreement. Furthermore, it is not compatible with any available COTS devices and networks.

Spectrum Agreement. SIFTs [3], part of WhiteFi, is a singlechannel bandwidth-independent signal detection algorithm used for determining the transmit bandwidth of an AP. FICA [24] uses binary amplitude modulation on multiple OFDM subcarriers, together with tight time synchronization, to enable each device to contend for different spectrum bands. Preamble detection on NC-OFDM networks [9] is useful for communications over disjoint spectral bands, but a separate mechanism must first be used to agree on the spectrum bands. Other typical uses for spectrum agreement include control channels [31] and backup channel lists [23].

\section{CONCLUSION}

We presented RodIN, a DSA extension platform that supports (a) direct connection to a COTS device, (b) fast FPGA-based spectrum shaping, and (c) I-FOP for fast and accurate spectrum agreement. A complete spectrum agreement and shaping operation can be carried out in about $10 \mu \mathrm{s}$, which adds only approximately $3.8 \%$ overhead to an $802.11 \mathrm{n}$ frame. We evaluated spectrum shaping and I-FOP using both simulations and real-world experiments, and demonstrated their efficacy even under low SIR levels.

\section{ACKNOWLEDGEMENTS}

We thank Suman Banerjee for shepherding our paper. We also thank Xinyu Zhang for the many insightful discussions on perframe spectrum shaping.

\section{REFERENCES}

[1] Linux ath9k driver. http://wireless.kernel.org/ en/users/Drivers/ath9k.

[2] Proposed TGac Draft Amendment (Draft 1.0) for IEEE 802.11 Wireless LANs, Jan. 2011.

[3] P. Bahl, R. Chandra, T. Moscibroda, R. Murty, and M. Welsh. White space networking with Wi-Fi like connectivity. SIGCOMM, 2009. 
[4] V. Brik, S. Banerjee, M. Gruteser, and S. Oh. Wireless device identification with radiometric signatures. MOBICOM, 2008.

[5] R. Chandra, R. Mahajan, T. Moscibroda, R. Raghavendra, and P. Bahl. A case for adapting channel width in wireless networks. SIGCOMM, 2008.

[6] C.-T. Chou, N. Sai Shankar, H. Kim, and K. G. Shin. What and how much to gain by spectrum agility? JSAC, 25(3), Apr. 2007.

[7] D. Chu. Polyphase codes with good periodic correlation properties. IEEE Trans. on Information Theory, 1972.

[8] L. Deek, E. Garcia-Villegas, E. Belding, S.-J. Lee, and K. Almeroth. The impact of channel bonding on 802.11n network management. In CoNEXT, 2011.

[9] A. Dutta, D. Saha, D. Grunwald, and D. Sicker. Practical implementation of blind synchronization in NC-OFDM based cognitive radio networks. In CoRoNet, 2010.

[10] S. Gollakota, F. Adib, and D. Katabi. Clearing the RF smog: Making 802.11 robust to cross-technology interference. SIGCOMM, 2011.

[11] R. Gummadi, D. Wetherall, and B. Greenstein. Understanding and mitigating the impact of RF interference on 802.11 networks. SIGCOMM, 2007.

[12] M. Heusse and F. Rousseau. Performance anomaly of 802.11b. In INFOCOM, 2003.

[13] S. Hong and S. Katti. DOF : A Local Wireless Information Plane. In SIGCOMM, 2011.

[14] S. Hong, J. Mehlman, and S. Katti. Picasso: Full Duplex Signal Shaping to Exploit Fragmented Spectrum. HotNets, 2011.

[15] V. Kone, L. Yang, X. Yang, B. Y. Zhao, and H. Zheng. On the feasibility of effective opportunistic spectrum access. IMC, 2010

[16] S. Lakshmanan, J. Lee, R. Etkin, S.-J. Lee, and R. Sivakumar. Realizing high performance multi-radio 802.11n wireless networks. In SECON, 2011.

[17] T. Lin and Y. Tseng. Collision analysis for a multi-Bluetooth picocells environment. IEEE Communications Letters, 7(10), 2003.

[18] M. Loiacono, J. Rosca, and W. Trappe. The snowball effect:
Detailing performance anomalies of 802.11 rate adaptation. In GLOBECOM, 2007.

[19] H. Rahul, N. Kushman, D. Katabi, C. Sodini, and F. Edalat. Learning to share: narrowband-friendly wideband networks. SIGCOMM, 38(4):147-158, 2008.

[20] S. Rayanchu, V. Shrivastava, S. Banerjee, and R. Chandra. FLUID: Improving throughputs in enterprise wireless LANs through flexible channelization. In MOBICOM, 2011.

[21] I. Selesnick, M. Lang, and C. Burrus. Constrained least square design of FIR filters without specified transition bands. IEEE Trans. on Signal Processing, 1996.

[22] V. Shrivastava, S. Rayanchu, J. Yoon, and S. Banerjee. 802.11n Under the Microscope. IMC, 2008.

[23] J. So and N. Vaidya. Multi-channel MAC for ad hoc networks: handling multi-channel hidden terminals using a single transceiver. In MOBICOM, 2004.

[24] K. Tan, J. Fang, Y. Zhang, S. Chen, L. Shi, and J. Zhang. Fine-grained channel access in wireless LAN. In SIGCOMM, 2010.

[25] K. Tan, Y. He, H. Shen, J. Zhang, and Y. Zhang. Spectrum virtualization layer. Technical Report MSR-TR-2011-108, Microsoft Research, 2011.

[26] K. Tan, J. Zhang, J. Fang, H. Liu, Y. Ye, S. Wang, Y. Zhang, H. Wu, W. Wang, and G. M. Voelker. Sora: High performance software radio using general purpose multi-core. In NSDI, 2009.

[27] USRP. http: / / www. ettus.com.

[28] WARP. http://mangocomm.com.

[29] M. Wellens and P. Mähönen. Lessons learned from an extensive spectrum occupancy measurement campaign and a stochastic duty cycle model. Mobile networks and applications, 2010.

[30] L. Yang, W. Hou, L. Cao, B. Zhao, and H. Zheng. Supporting demanding wireless applications with frequency-agile radios. NSDI, 2010.

[31] Y. Yuan, P. Bahl, R. Chandra, and P. Chou. Knows: Kognitiv networking over white spaces. DySPAN, 2007.

[32] X. Zhang and K. G. Shin. Adaptive subcarrier nulling: Enabling partial spectrum sharing in wireless LANs. ICNP, 2011. 